\title{
Results from the Mediterranean neutrino detectors
}

\author{
The KM3NeT and ANTARES Collaborations ${ }^{\ddagger *}$ \\ \$ https://www.km3net.org/km3net-author-list-for-icrc-2019 \\ * http://antares.in2p3.fr/Collaboration/index2.html \\ E-mail: coniglionedlns.infn.it
}

Since 2007 the ANTARES high energy neutrino detector, installed in the depths of the Mediterranean Sea, is taking data. It is located $40 \mathrm{~km}$ off the French coast instrumenting a volume of about 0.01 cubic kilometers. The main aim of the ANTARES neutrino telescope is the search of astrophysical neutrinos as a diffuse flux or coming from astrophysical sources. ANTARES can observe in real time neutrinos correlated in space and time with other cosmic probes (multimessenger approach) providing valuable informations from the Northern sky.

With the aim to enlarge the effective areas for the detection of high energy neutrinos and also to give valuable responses in the neutrino physics domain, the KM3NeT collaboration is constructing two detectors in the Mediterranean Sea: KM3NeT/ARCA mainly devoted to the discovery and observation of neutrinos from Active Galactic Nuclei and Galactic sources and KM3NeT/ORCA devoted to the study of the fundamental properties of the neutrino.

In this work the main recent results achieved by the ANTARES collaboration and the KM3NeT perspectives as well the first results from the deployed KM3NeT Detection Units will be reported.

Corresponding author: Rosa Coniglione ${ }^{\dagger 1}$

${ }^{1}$ INFN - Laboratori Nazionali del Sud (LNS), Via Santa Sofia 62, 95123 Catania, Italy

36th International Cosmic Ray Conference -IceCubeRC2019-

July 24th - August 1st, 2019

Madison, WI, U.S.A.

\footnotetext{
${ }^{*}$ for collaboration list see PoS(ICRC2019)1177

†speaker.
} 


\section{Introduction}

The study of astrophysical high-energy neutrinos can add relevant pieces of information to the understanding of production mechanisms of primary cosmic rays (CRs) and high energy $\gamma$ rays. Neutrinos, CR and high energy $\gamma$-rays can be produced at the sources or in the encountered dense source environment. Unlike CRs, which in their path towards the Earth are deflected by the intergalactic magnetic fields or absorbed as they interact with matter or radiation via inelastic collisions, neutrinos travel undisturbed. High energy $\gamma$-rays $(\mathrm{E}>300 \mathrm{TeV})$ are absorbed by cosmic microwave background radiation. Only by combing information from all three messengers, will we be able to to achieve a complete picture of non thermal processes in the Universe. Recently also Gravitational Waves have been added to the number of probes that can be associated to energetic phenomena in the cosmos.

The existence of neutrinos of cosmic origin has been demonstrated by IceCube with the detection of a diffuse flux exceeding the expected background of more than 5 sigma. The origin of the bulk of these neutrinos from the full sky is yet unknown. Only thanks to the simultaneous observation with other astrophysical messengers the first neutrino source has been discovered [1,2]. This, together with the observation of counterparts of a gravitational waves source [3], has confirmed that multi-messenger astronomy is the future key objective of the astrophysical and astronomical communities.

Mediterranean Sea offers an optimal location for the observation of the Southern sky, where the Galactic center and most of the Galactic Plane are located. The ANTARES telescope [4] and the future KM3NeT detectors [5] offer a unique opportunity to observe the neutrino sky from a complmentary field of view w.r.t. IceCube. Thanks to the optical water properties, they provide an unprecedented angular resolution, which is a crucial parameter for the pointing of neutrino sources. KM3NeT will be a distributed infrastructure comprising two telescopes: ARCA (Astroparticle Research with Cosmic in the Abyss), located at a depth of $3500 \mathrm{~m}$ offshore Capo Passero (Italy), and ORCA (Oscillation Research with Cosmic in the Abyss), located at a depth of $2500 \mathrm{~m}$ offshore Toulon (France). The KM3NeT/ARCA detector, optimized for the neutrino energy range from $100 \mathrm{GeV}$ to $10^{8} \mathrm{GeV}$, focuses on the high-energy neutrino astrophysics while the KM3NeT/ORCA detector, optimized for neutrino energies from few $\mathrm{GeV}$ to $100 \mathrm{GeV}$, focuses on the study of fundamental neutrino properties. With the two detectors KM3NeT will cover a very wide range of neutrino energies with high effective areas.

The ANTARES results collected in 11 years of data taking cover many aspects of the neutrino physics. Currently, ANTARES is providing an active real-time monitoring of the Southern Sky with the best sensitivity in the 1-300 $\mathrm{TeV}$ domain. This is of fundamental importance for the follow-up studies of astrophysical transients including also gravitational waves.

The detectors will be described in Section 2. The recent results achieved in the searches for point-like sources also in a joint analysis with IceCube will be reported in Section 3 together with the perspectives for the future KM3NeT/ARCA detector. The search of neutrinos from the all sky and from extended regions proposed as hadronic acceleration sites will be shown in Section 4. The results from transient sources and the alert system of ANTARES will be covered in Section 5. The indirect search for dark matter is also one of the main results of the collaboration and results will be presented in Section 6. Results from neutrino oscillation studies and perspectives are reported 
in Section 7. Finally in Section 8 the KM3NeT construction status will be presented together with the first results obtained.

\section{The Detectors}

ANTARES [4], has been the first operating deep sea neutrino detector. It is installed at $42^{\circ} 48^{\prime}$ $\mathrm{N}, 6^{\circ} 10^{\prime} \mathrm{E}$ at about $40 \mathrm{~km}$ off shore the French coast at a depth of about $2500 \mathrm{~m}$, and is continuously taking data in its complete configuration since May 2008 with a duty cycle of about $95 \%$. ANTARES consists of about 885 optical modules, each one housing a 10-inch photomultiplier (PMT), arranged in groups of three (a storey) and distributed along 12 vertical strings. The PMTs of each storey are facing $45^{\circ}$ downwards to optimize the detection of light for up-going neutrinos. The strings are anchored on the sea floor, arranged in an octagonal configuration with a inter-string distance of about $60 \mathrm{~m}$, and kept vertical by a submerged buoy. The total volume of the ANTARES detector is of about $0.01 \mathrm{~km}^{3}$.

With the aim to enlarge the effective areas for the detection of high energy neutrinos and to study the neutrino oscillation properties detecting atmospheric neutrino, the KM3NeT collaboration has started the construction of two detectors: ARCA (Astroparticle Research with Cosmics in the Abyss) and the ORCA (Oscillation Research with Cosmic in the Abyss) detectors [5].

An innovative optical sensor, the Digital Optical Modules (DOM), has been developed by the KM3NeT collaboration. Each DOM is a 17-inch diameter pressure-resistant glass sphere housing 31 3-inch PMTs together with the associated electronics. The lower hemisphere of each DOM contains 19 PMTs, which are thus downward-looking, whereas the other 12 PMTs look upwards. Eighteen DOMs are suspended by means of two ropes along a vertical structure, called the Detection Unit (DU). The DUs are flexible structures anchored to the sea floor and kept almost vertical by submerged buoys. An electro-optical backbone cable, running along the full length of each DU, allows for power feeding and data transmission. Each DU is connected at its base to a sea floor network of electro-optical cables and junction boxes, which converges in a single main electrooptical cable connecting to shore. The readout is based on the "all-data-to-shore" concept in which all analogue signals from the PMTs that pass a preset threshold (typically 0.3 photo-electrons) are digitised and all digital data are sent to shore where they are processed in real time. To simplify its integration and deployment, each DU is wrapped on a $2.5 \mathrm{~m}$ diameter spherical frame which is deposited on the seabed. The DU is then unfurled until it reaches its operating configuration. An array of 115 DUs will constitute a detector building block.

The ARCA and ORCA detectors will share the same technology for the construction and deployment of the main components of the detectors, although with different granularity. ARCA detector will comprise two building blocks (230 DUs) with DUs horizontally spaced by about 90 $\mathrm{m}$ and with a vertical spacing between DOMs of about $36 \mathrm{~m}$ for a total volume of about $1 \mathrm{~km}^{3}$. It will be installed at $36^{\circ} 16^{\prime} \mathrm{N} 16^{\circ} 06^{\prime} \mathrm{E}$ at about $80 \mathrm{~km}$ off-shore the Sicilian coast in front of Capo Passero, Italy, at a depth of $3500 \mathrm{~m}$. ORCA will comprise one building blocks (115 DUs) with DOMs vertically spaced on average by about $9 \mathrm{~m}$ and with DUs with an horizontal distance of about $20 \mathrm{~m}$. The detector instruments about 8 Mton of sea water. It will be located at $42^{\circ} 48^{\prime} \mathrm{N}$ $06^{\circ} 02^{\prime} \mathrm{E}$ at about $40 \mathrm{~km}$ off the coast of Toulon, France, at a depth of about $2450 \mathrm{~m}$. The ORCA detector is located at about $10 \mathrm{~km}$ west the site of ANTARES. 


\section{Point-like neutrino searches}

The search for Galactic and extragalactic neutrino sources is one of the primary goals of an high energy neutrino telescope. Different type of astrophysical objects have been proposed as acceleration sites able to produce a neutrino flux through hadronic interactions. In ANTARES the search for neutrino sources has been performed following several approaches.

The ANTARES 9 years analysis presented in Ref.[6] has been recently updated adding two more years of data (up to December 2017) for a total live time of 3125 days [7]. In the full sky search all the sky in the field of view of ANTARES is scanned in $1^{\circ} \times 1^{\circ}$ bins to search for clusters of neutrinos. For each bin the significance, based on the likelihoods of the signal and background, is estimated. The point in the sky with the higher significance remains unchanged at declination $\delta=+23.6^{\circ}$ and right ascension $\alpha=343.5^{\circ}$. No new events in the sample of data from 2015 to 2017 was found and the pre-trial value of $1.510^{-6}$ and a post-trial value of 0.23 corresponding to $1.2 \sigma$ was assigned. Around the most significant direction $18(3)$ tracks within $5^{\circ}\left(1^{\circ}\right)$ and 1 shower event within $5^{\circ}$ were found. The same position in the sky was found with the same pre-trial value when the directions of 75 IceCube track neutrino candidates [7] were investigated.

In Fig.1 the upper limit for the selected sources and the sensitivity, assuming a spectrum with a $\mathrm{E}^{-2}$ dependence, are shown as a function of the source declination. Also reported (green line) is the sensitivity of the KM3NeT/ARCA detector, that will allow for an increase of a factor 20 after 6 years of observation. The KM3NeT/ARCA detector thanks to the high sensitivity and to the optimal angular resolution $\left(<0.1^{\circ}\right.$ at $1 \mathrm{PeV}$ [5]) is the optimal detector to look for extragalactic sources in a wide field of view.

An alternative approach is to search in the directions of a pre-selected list of candidate sources. This analysis has been presented in Ref. [6] and more recently updated with the addition of 5 more sources, recently observed, reaching a total of 111 Galactic and extragalactic candidate sources. The highest number of cluster events was found at the location of HESSJ0632+057 (at $(\alpha, \delta)$ $\left.=\left(98.24^{\circ}, 5.81^{\circ}\right)\right)$ unchanged w.r.t. the analysis in Ref.[6]. The post-trial value is of $16 \%(1.4 \sigma)$.

The neutrino source detected by IceCube, the blazar TXS0506+056, was also investigated in steady mode with 9 years of data and time-dependent search and the results are reported in Ref.[8]. No excess of events was found in both analyses and upper limits in the flux were set. In the steady mode search adding two more years of ANTARES data no further events were found in the direction of the blazar and upper limits were set assuming different spectral indexes.

To increase the significance that would be observed for a single source, a stacking analysis has been performed [9] using 11 years of ANTARES data. The different source populations considered are: Fermi3LAC catalog containing 1420 blazar, a sub-sample of BL Lacs (604 sources), 414 FSRQ, 15 Dust obscured AGN and 65 Radio-Galaxies. Moreover, 56 IceCube events in the field of view of ANTARES were also considered. The results of this analysis has shown that the most significant population is represented by the Radio-Galaxies with a post-trial significance of $1.6 \sigma$, where all the sources are assumed to have the same flux. The most significant source among the FSRQ population is the 3C403 with a chance to be casually found among the 56 Radio-Galaxies of $2.5 \sigma$. Another interesting source among the Fermi 3LAC BL Lacs population is the MG3J225517+2409 that has been measured by Fermi (corresponding to Fermi 3FG J2255.1+2411) and classified as BL Lac and LSP (Low Synchrotron Peak) and with unknown red- 

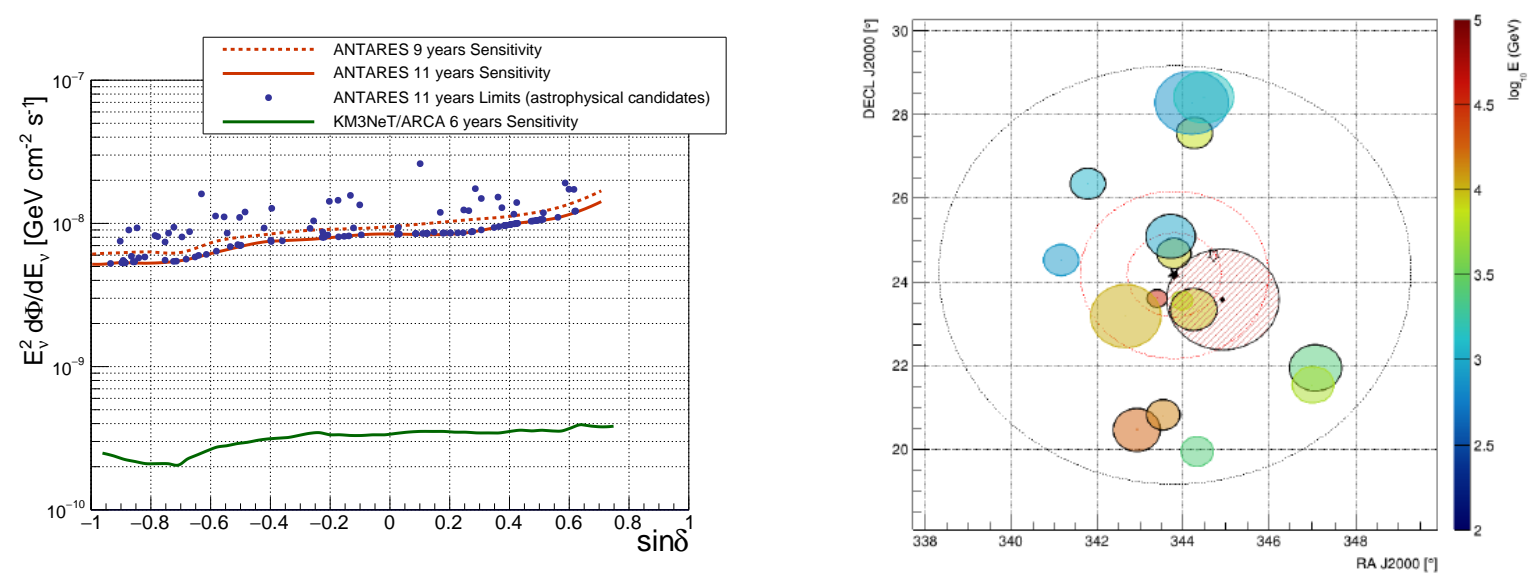

Figure 1: Left - ANTARES upper limits for the investigated astrophysical candidates (blue dots) as a function of the source declination. The red solid line shows the median sensitivity. For comparison, the curve from the nine years ANTARES point-like source analysis [6] (red dashed line) and the 6 years sensitivity for KM3NeT/ARCA (green solid line) are also reported. Right - Blazar MG3 J225517+2409. Each event is represented by a filled circle with a radius equal to its angular error estimate. The estimated energy of the event is indicated by the color code. The concentric circles (dashed lines) centered around the source (black star marker) indicate the $1^{\circ}, 2^{\circ}$ (red) and $5^{\circ}$ (black) angular distances to the source. The nearby Fermi source 3FGL J2258.8+2437 is indicated as a empty star marker, and the position of the IceCube track by the red-hashed circle with a black diamond marker. The ANTARES events that do have arrival times within the range of the available Fermi blazar light curve are circled by a bold black line.

shift. Five ANTARES events were found within $1^{\circ}$ from the location of this source (Fig. 1(right)). The IceCube EHE ID3 [10] lies at a distance of $1.1^{\circ}$. Without including the IceCube ID3 event the pre-trial probability is $1.410^{-4}(3.8 \sigma)$. The probability to found by chance a similar excess among the 1255 blazars is $0.16(1.4 \sigma)$. To explore more in details this source the emission light curve for $\gamma$-rays above $100 \mathrm{MeV}$ has been analyzed. It was found that in a 5 months period (around July 2010) the intensity has increased of about a factor 5. No ANTARES events were found in the flaring period while the IceCube EHE ID3 is detected inside the flaring period. From a time dependent analysis combining the ANTARES and IceCube p-values, assuming that the neutrino flux is produced continuously by the source, a global pre-trial value of $2.210^{-7}(5.2 \sigma)$ was found (for more details see [9]).

The events in the Southern sky resulting from the ANTARES nine-years analysis [6] and those of the IceCube seven-years analysis [11] were joined and analyzed to increase the sensitivity for the search in the Southern sky [12]. With this sample of events different searches for cosmic neutrinos were performed. In the full sky search the most significant point in the Southern sky has coordinates $(\alpha, \delta)=\left(213.51^{\circ},-40.798^{\circ}\right)$, extension $0^{\circ}$ and post trial value of 0.18 . Moreover, 57 preselected known cosmic objects were selected and a search in these directions performed. No excess was found and upper limits were set [12]. This joined analysis increased of $30 \%$ the sensitivity of ANTARES that, despite the limited size, remains the best neutrino telescope for the search of neutrinos from point-like sources in the Southern sky.

In Tab. 1 the most significant sources identified in the different ANTARES analyses are re- 


\begin{tabular}{|c|c|c|c|cc|} 
Analysis & Source & $\alpha\left[^{\circ}\right]$ & $\delta\left[^{\circ}\right]$ & pre-trial $(\sigma)$ & post-trial $(\sigma)$ \\
\hline full sky & & 343.5 & +23.6 & $1.510^{-6}(4.8)$ & $0.23(1.2)$ \\
candidate list & HESSJ0632+057 & 98.24 & +5.81 & $1.510^{-2}(2.4)$ & $0.16(1.4)$ \\
IceCube tracks & EHE ID3 & 343.5 & +23.6 & $1.510^{-6}(4.8)$ & $0.015(2.4)$ \\
TXS0506+056 & & 77.36 & +5.69 & $3.410^{-2}(2.1)$ & $0.87(0.16)$ \\
ANT-IceCube Southern sky & & 213.2 & -40.8 & $1.310^{-5}(4.3)$ & $0.18(1.3)$ \\
ANT-IceCube RXJ1713 & & 258.25 & -39.75 & $4.010^{-1}(0.84)$ & \\
stacking Radio-galaxies & & - & - & $4.810^{-3}(2.8)$ & $0.10(1.6)$ \\
stacking Radio-galaxies & & 298.06 & +2.5 & $2.310^{-4}(3.7)$ & $0.013^{*}(2.5)$ \\
stacking 3LAC BL Lacs & $3 C 403$ & - & - & $8.810^{-2}(1.7)$ & $0.64(0.5)$ \\
stacking 3LAC BL Lacs & MG3J225517+2409 & 343.78 & +24.19 & $1.410^{-4}(3.8)$ & $0.16^{*}(1.4)$ \\
Time Analysis & MG3J225517+2409 & 343.78 & +24.19 & $1.410^{-4}(3.7)$ & $0.16^{*}(1.4)$ \\
Time Analysis ANT-IceCube & MG3J225517+2409 & 343.78 & +24.19 & $2.210^{-7}(5.2)$ & -
\end{tabular}

Table 1: Results from the ANTARES point-like search analyses. For each analysis the most significant point in the sky, with declination, $\delta$, and right ascension, $\alpha$, as well the pre-trial and post-trial values, are reported. The significance (two-side convention) is also indicated. A $\mathrm{E}^{-2}$ spectrum is assumed in all the analyses except for the RXJ1713 ANTARES-IceCube combined analysis (ANT-IceCube) that was performed assuming a neutrino energy spectrum with slope 2.06 and energy cutoff of $8.04 \mathrm{TeV}$ (see [12]). The post-trial values with $\mathrm{a}^{*}$ are evaluated with the formula reported in [9].

ported together with the pre and post-trial probabilities and the correspondent sigma value in the two-sided convention.

The detection of neutrino from Galactic sources is challenging for a neutrino telescope because of their spacial extension and the presence of an energy cutoff in the $\mathrm{TeV}$ range in the expected neutrino energy spectrum. The most promising neutrino Galactic candidate sources are the SNR. These are intense emitters of high energy $\gamma$-rays and sites of proton acceleration as evidenced in 2013 by Fermi-LAT observations of the SNRs W44 and IceCube 443 [13]. The sensitivity of the KM3NeT/ARCA neutrino telescope to Galactic neutrino sources has been evaluated [14] for a selection of sources from the TeVCat catalogue. The $3 \sigma$ discovery potential for several Galactic sources is reported in Fig. 2(left). The SNR RXJ1713.7-3946 can be observed with a significance of $3 \sigma$ within 5.5 years. Stacking the two most intense SNR sources the RXJ1713.7-3946 and the Vela Jr. the $3 \sigma$ observation can be achieved in 2.5 years (Fig. 2(right)).

\section{Diffuse neutrino searches}

Observed with statistically high significance in several IceCube analyses, a diffuse flux of cosmic neutrinos can be now considered as a guaranteed flux. The results obtained by IceCube for up-going tracks, that mainly look for neutrinos from the Northern Hemisphere [10], has evidenced a flux with a slope $\Gamma=2.13 \pm 0.13$ while in the analysis where high energy events are selected from all sky (mainly cascade events) a softer spectrum was evidenced ( $\Gamma=2.5 \pm 0.09)$ [15] and even softer $\left(\Gamma=2.9_{-3.9}^{+0.33}\right)$ in [16]. No point-sources have been associated to date and since candidate source populations, as AGN and GRB cannot explain the observed excess, a high number of weak sources was suggested as an explanation. [17]. An alternative explanation is based on a two-component 

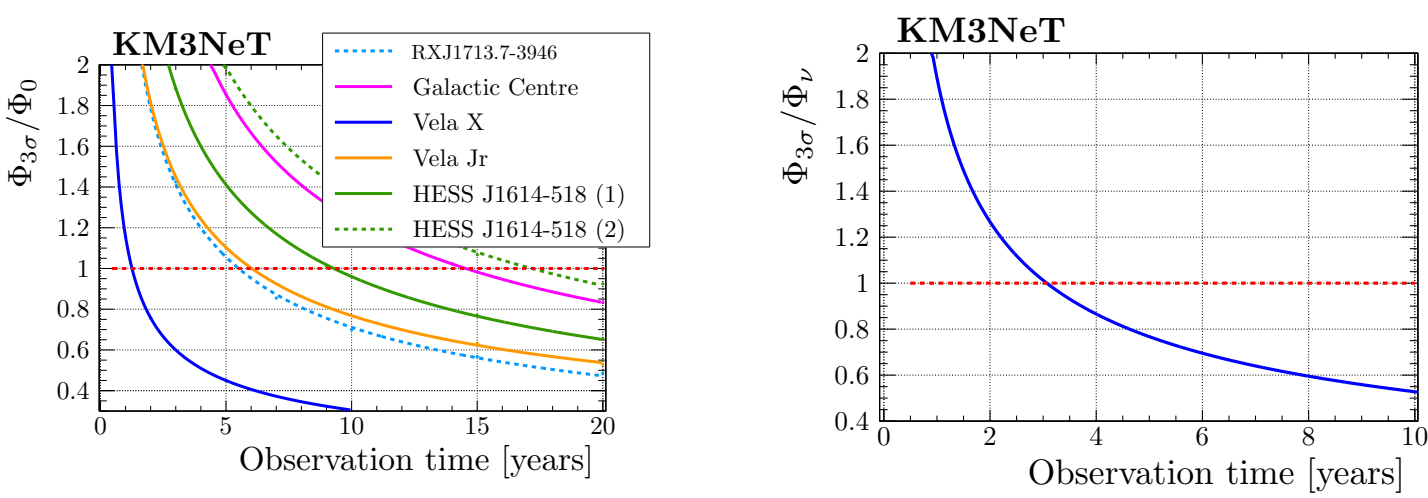

Figure 2: Ratio of the discovery potential for KM3NeT/ARCA $\Phi_{3 \sigma}$ to the expectation flux $\Phi_{v}$ as a function of the observation time for the sources reported in the legend (right) and for the stacking analysis including RX J1713.7-3946 and Vela Jr (right).

spectrum, with a softer diffuse component of Galactic origin and a harder one originating from extragalactic sources [18].
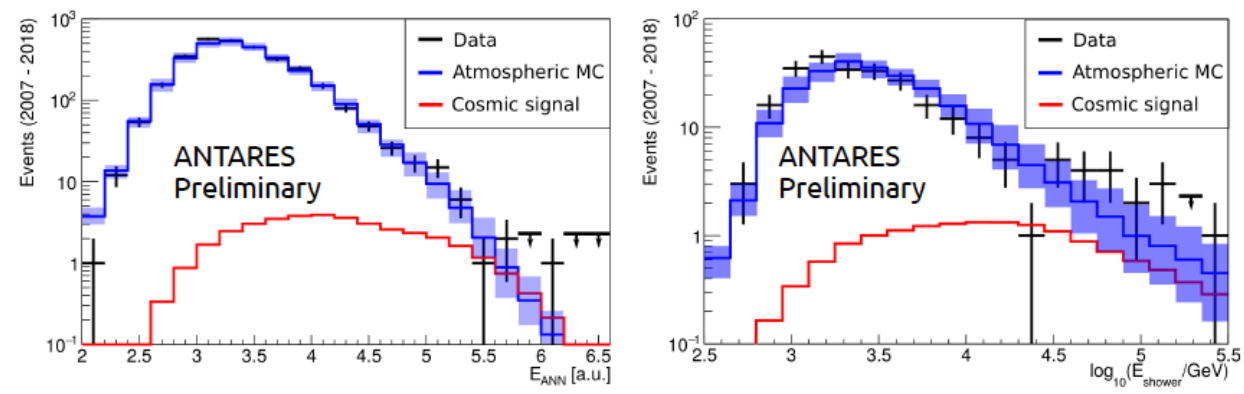

Figure 3: Energy estimator distributions for track-like events (left) and shower-like events (right) for the selected events. The red line shows the cosmic flux as estimated in this analysis and the blue line represents the background contribution. The blue band represents the systematic uncertainties of the MC background simulation.

ANTARES has already observed a small excess of neutrino with a significance of $1.6 \sigma$ in the analysis of 9 years of data [19]. Recently the data set has been extended [20] adding data collected from January 2016 to June 2018 for a total livetime of 3350 days. The analysis, based on the analysis of track and shower events, has selected 50 events ( 27 track-like events and 23 shower-like events) as cosmic neutrino candidates. The Monte Carlo expectation for the background, simulated assuming the Honda [21] model for the conventional component and the Enberg et al. [22] model for the prompt component, is of $36.1 \pm 8.7$ events. In Fig. 3 the distribution for the energy estimator for track like events (left) and the shower-like events (right) are reported. The excess observed has a significance of $1.8 \sigma$. For the cosmic flux the best fit parameters obtained for an unbroken power law spectrum are: $\Gamma=2.3_{-0.4}^{+0.4}$ and $\Phi_{0}(100 \mathrm{TeV})=1.5 \pm 1.010^{-18} \mathrm{GeV}^{-1} \mathrm{~cm}^{-2} \mathrm{~s}^{-1} \mathrm{sr}^{-1}$.

This spectrum is reported in Fig. 4(left) (red band) together with the IceCube diffuse fluxes from two different analyses. The red line in Fig. 4(left) is the diffuse flux spectrum assumed in the KM3NeT/ARCA analysis for the estimate of the number of years needed to observe an excess at 
different significance levels that is reported in Fig. 4(right). This estimate, based on combined track and cascade events, has been calculated for two different detector configurations: the complete detector made of 2 building blocks (330 DUs) and only one building block (115 DUs). A $5 \sigma$ level can be reached in about 1 year of data taking with the first block of 115DUs.
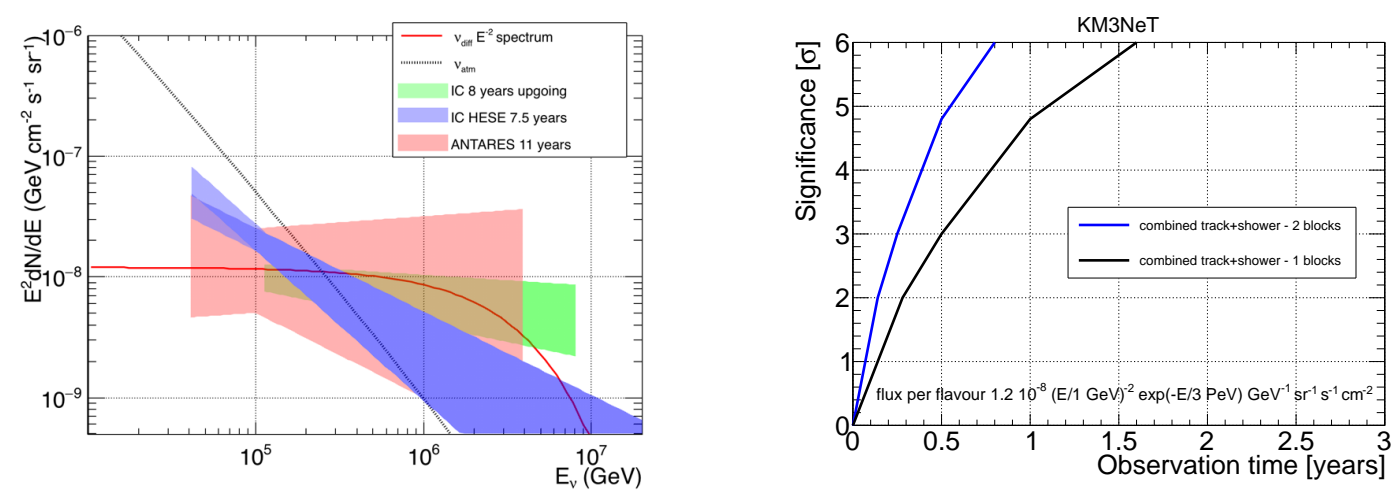

Figure 4: Left - Diffuse signal fluxes as a function of the neutrino energy. The blue band represents the flux estimated from the IceCube HESE analysis [16], the green band from the IceCube 8 years up-going track analysis [23] and the red band from the ANTARES 11 years analysis [20]. The red line represents the diffuse flux assumed in the KM3NeT LoI [5]. The atmospheric neutrino flux is also reported (black dashed line). Right - Significance as a function of time for the detection of a diffuse flux of neutrinos corresponding to the red line in the right figure, for cascade-like and track-like combined events. The blue line is for the full ARCA detector ( 2 building blocks - 330 DUs) and the black line for one building block (115 DUs).

The region around the Galactic Plane is an interesting region accessible with high visibility by a neutrino telescope located in the Mediterranean Sea. Diffuse flux from the Galactic plane and in particular near the Galactic Center is expected to be enhanced due to a larger matter density. Constraints on the emission from the Galactic plane has been set from ANTARES [24] and more recently in a joint analysis with IceCube data [25]. In this analysis the diffuse emission from the Galactic plane estimated with the KRA model [26] has been constrained. In the KRA model a nonuniform cosmic-ray (CR) transport scenario with a radially dependent diffusion coefficient has been adopted to explain the high-energy diffuse $\gamma$-ray emission along the whole Galactic Plane, as well as the hardening of $\mathrm{CR}$ spectra measured at $250 \mathrm{GeV}$ and the possible cutoffs of Galactic CRs. The neutrino signal, $\Phi_{K R A}$, from this region has been tested assuming a $5 \mathrm{PeV}$ and $50 \mathrm{PeV}$ cutoffs for CR primaries. No excess of events has been observed and the upper limits have excluded a neutrino flux with a normalization factor of about $1.1 \times \Phi_{K R A}$ at $90 \%$ confidence level.

To explain the astrophysical high energy neutrino flux observed by IceCube, neutrino emission from the Local superbubble wall was hypothesized [27]. The bubbles are large regions of the sky originated by the stellar wind from massive stars that expanding collides with the gas of interstellar medium creating an expanding low density bubble. The wall of the superbubble presents higher matter density and higher magnetic fields. The Sun is located inside a superbubble called Local superbubble that overlaps with the nearby Loop I superbubble forming a wall of dense material. Cosmic rays generated by sources inside the superbubble can interact with the wall region and generated neutrino. A search for neutrinos from the Loop1 region has been carried out using data collected by the ANTARES neutrino telescope over 11 years of data acquisition [28]. No excess 
has been found and an upper limit 6.5 times larger than the reference flux was set.

The region around the Galactic Plane will also be a target for KM3NeT/ARCA. The expected sensitivities for this region are really promising. The expectations for a diffuse high-energy neutrino flux from a Galactic Plane region near the Galactic Center $\left(|\ell|<30^{\circ}\right.$ and $\left.|b|<4^{\circ}\right)$ [29] have been reported for the track channel and for the cascade channel. In both the channels after four years of observation the neutrino flux predicted in the KRA model [26] can be discovered with a large significance. The Low Latitude Bubble (LLB) region $\left(|\ell|<15^{\circ}\right.$ and $|b|<10^{\circ}$ ) of the known Fermi Bubbles was also considered [30] as the expected neutrino flux from this region has a higher cutoff w.r.t the neutrino flux from the entire bubbles region. Considering only up-going track-like events it has been estimated that the expected flux will be observable with 5 years of data of one building block of KM3NeT/ARCA, even considering the case of a pessimistic cutoff at $100 \mathrm{TeV}$.

\section{Multimessenger and transient sources}

The simultaneous observation in space and time by neutrino telescopes, gravitational waves interferometers and multi-wave detectors (multi-messanger astronomy) is and will be in the next future the key approach for the understanding of the most remote and extreme phenomena in the cosmos.

ANTARES has implemented since 2009 a follow-up program of neutrino alerts [31]. This alert system consists of real-time triggers and an on-line track reconstruction algorithm. The triggers currently implemented are based on the detection of one following events: a single high energy neutrino (HE); a very high energy (VHE) neutrino; a neutrino associated with specific directions in the sky; at least two neutrinos coming from close directions. When one event satisfies at least one of these criteria, an alert is issued to robotic optical telescopes (MASTER, TAROT, ROTSE, ZDKO and SWOM) which immediately start the observation and schedule the observation in the following weeks. About 25 alerts per year are sent to these optical ground based telescopes. A fraction of them (about 6 per year) that satisfy the VHE trigger are also sent to the MWA low-frequency radio satellites, to the Swift and INTEGRAL X-ray/ $\gamma$-ray satellites and to the H.E.S.S. high-energy $\gamma$ ray telescope. The system is able to send in about $6 \mathrm{~s}$ alerts for events with a resolution of about $0.5^{\circ}$. More than 300 alerts have been issued since 2009 and no counterpart detected in the different searches [32].

The real time searches of neutrino candidates in coincidence with Swift, Fermi, IceCube high energy neutrinos and Gravitational Wave (GW) events performed with the ANTARES detector are presented in [33]. These real-time analyses have been developed to react in short time to the public alerts sent by the Gamma-ray Coordinated Network (GCN) or by private alerts transmitted through special channels. Sources of neutrinos and GW have been searched from the $\mathrm{O} 2 \mathrm{Ligo/Virgo}$ run with the following strategy: temporal coincidence between the $\pm 500 \mathrm{~s}$ and \pm 1 hour around the GW alert and a spacial overlap between the $90 \%$ GW probability contour and the ANTARES visibility region at the time of GW alert [34]. Neutrinos from sub-threshold events (events with low signal to noise ratio) from Ligo $\mathrm{O} 1$ run were searched recently using HEN events detected by ANTARES and IceCube collaborations [35]. No counterpart has been detected until now. Also for the binary neutron star merger GW170817, for which electromagnetic counterparts have been observed, no 
neutrino counterpart was found by the ANTARES and IceCube neutrino telescopes and the Pierre Auger Observatory [3].

Since 2016 IceCube also sends alerts when a high-energy starting events (HESE) or extremely high energy track candidates (EHE) are observed. The ANTARES strategy for the follow up is, in this case, too look for each IceCube alert whose position is below the horizon for ANTARES at the time of the alert and inside a cone of $3^{\circ}$ around the IceCube alert coordinate. Up to now IceCube has issued 27 alerts, 11 of which have been followed by ANTARES without any detection [33].

From the experience gained in ANTARES the implementation of an alert system in KM3NeT has already started. In particular the online reconstruction has been already implemented in both sites and studies for the setting of specific triggers started. In particular a study for the detection of Core-collapse SuperNovae (CCSN) has started and the online trigger implemented. CCSN are among the most energetic phenomena in the Universe. They are intense transient sources of $\mathrm{MeV}$ neutrinos. The optical outburst begins only hours after the actual onset of the explosion in the very center of the star and neutrinos are a unique probe to get direct and immediate information about the supernova "engine". A study to prove that KM3NeT, thanks to the DOM structure, is able to detect MeV neutrinos from CCSN explosion has been reported in [36]. With MC simulations and with the data collected with the first strings deployed a technique that, in each single DOM, selects hits from CCSN signal from the backgrounds has been developed. The backgrounds in this context are hits from ${ }^{40} \mathrm{~K}$ decay and from atmospheric muons. The number of PMTs in a DOM with hits in a time window of 10ns is called "multiplicity" (M). Data analysis reported in [37] has shown that high values of $\mathrm{M}$ are due to atmospheric muons while low values of $\mathrm{M}$ are due to ${ }^{40} \mathrm{~K}$ decay. After the rejection of atmospheric muons the multiplicity distribution is reported in Fig. 5(left). The atmospheric muon rejection is operated selecting coincidence between different DOMs with $\mathrm{M}>4$ at the microsecond scale. The contribution of hits from ${ }^{40} K$ is negligible for $\mathrm{M}>3$. As shown in Fig. 5(left), where also the signal expectation is reported for two different hypothesis of mass progenitors at $10 \mathrm{kpc}$, the multiplicity range where the signal is expected is in the range of $6 \leq \mathrm{M} \leq$ 10. If we take into account all the DOMs in the detector a detection significance can be evaluated for a fixed progenitor mass as a function of the CCSN distance. Combining the two complete KM3NeT detectors, for a total of about 6000 DOMs, a significance of $5 \sigma$ is achieved for a CCSN of $27 \mathrm{M}_{\odot}$ at 25kpc (Fig. 5(right)).

An online trigger, based on the number of DOMs detecting a coincidence and passing the $\mathrm{M}>4$ selection, has been developed for the generation of real-time alerts and the participation at the SNEWS network [36]. This trigger is already implemented and will assure, when ARCA and ORCA will be competed, the participation to the SNEWS network with the capability to detect a Galactic CCSN of mass higher than $27 \mathrm{M}_{\odot}$.

\section{Indirect dark matter searches}

Consensus on the existence of dark matter is now shared between different communities but its existence has not yet been proved. The experimental techniques are grouped in two main classes: the direct search and the indirect searches. Indirect experiments search for annihilation or decay of dark matter contributing to charged cosmic rays, photons in a wide range of energies, and neutrinos. In particular, neutrino telescopes are good tools for the search of neutrinos produced in 

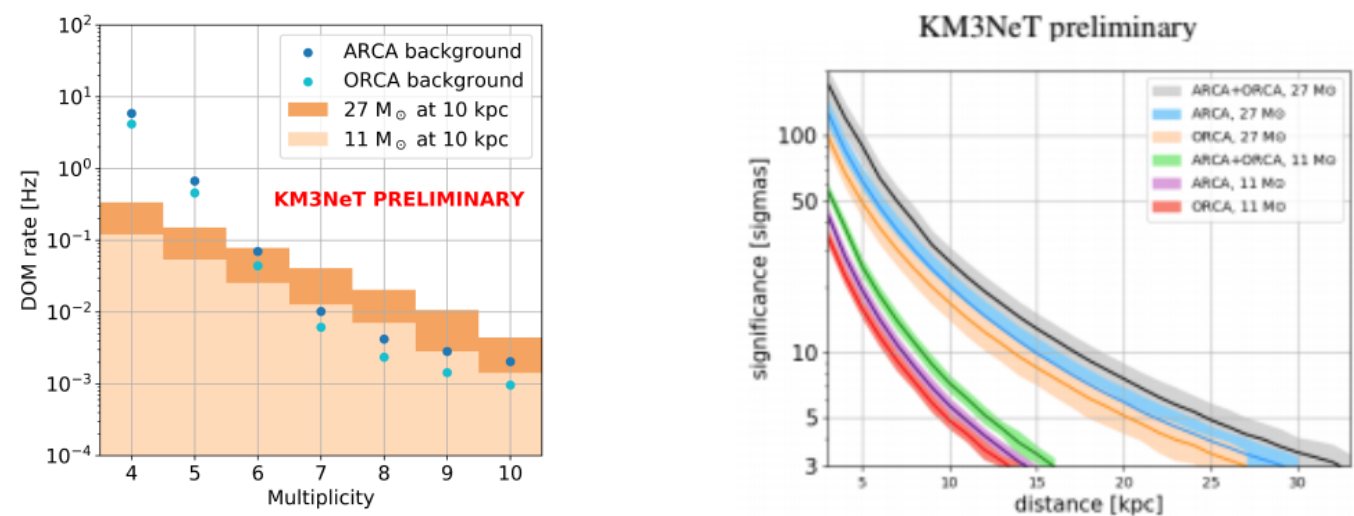

Figure 5: Left - DOM rates after muon rejection as a function of the multiplicity for background and signal expected for a $27 \mathrm{M}_{\odot}$ and $11 \mathrm{M}_{\odot}$ progenitors at 10kpc. Right - Detection significance for ORCA and ARCA detectors as a function of the CCSN distance.

the annihilation of Weakly Interacting Particles (WIMPs) accumulated in cosmic regions with high density such as the Galactic Center (GC) and the Sun.

Track events collected in 11 years of ANTARES data have been analyzed to search for neutrinos originated in the annihilation of WIMPs in the Galactic Center [38]. The search was done selecting the highest-yield channels of annihilation of WIMPs that lead directly or indirectly to neutrinos. The channel selected are: $b \bar{b}, \tau^{+} \tau^{-}, W^{+} W^{-}, \mu^{+} \mu^{-}, v \bar{v}$ and the neutrino energy spectra for each annihilation and for each annihilation channel are taken from [39]. The neutrino energy spectra depend on the assumed WIMP mass, constrained in this analysis to a maximum value of 100 $\mathrm{TeV}$. Moreover, predictions of the neutrino fluxes deriving from dark matter annihilation strongly depends on the parameterization of the dark matter density. In our analysis three halo models are considered (NFW, Burkert, McMillan) and the most stringent limit is obtained for the $v \bar{v}$ channel and the NFW halo model. In Fig. 6 limits on the thermally averaged cross-section for WIMP pair annihilation set with 11 years of data for the $\tau^{+} \tau^{-}$channel are reported. This limit is compared with limits of $\gamma$-ray detectors (the $\tau^{+} \tau^{-}$channel yields as final product also $\gamma-\gamma$ ). At high WIMP masses the most stringent limits are that one of HESS collected in 10 years of observation. The IceCube upper limit was obtained with 3 years of IceCube86/DeepCore [40] data. Very competitive limits are expected for ARCA full detector (230 DUs) after one year of observation as shown in Fig. 6(left) where the sensitivity is reported (dashed red line).

An effort to unify the analysis methods and the expected neutrino spectra was done in the combined search for neutrinos from the Galactic Center with ANTARES and IceCube data [41]. The upper limit, obtained from ANTARES [42] and IceCube [40] track events, is shown in Fig. 6(right) for all the channels analyzed and the NFW halo model. The first combined limits show improvements for the WIMP mass range from $50 \mathrm{GeV}$ to $1 \mathrm{TeV}$. Further improvements are expected selecting different data samples and improving the analysis method.

Limits for Dark Matter from the Sun has been set by ANTARES [43] and recently also first ORCA sensitivity have been estimated [44] for the range of WIMP masses from 15 to $100 \mathrm{GeV}$ showing that the ORCA detector after only one year of data is a competitive detector. 

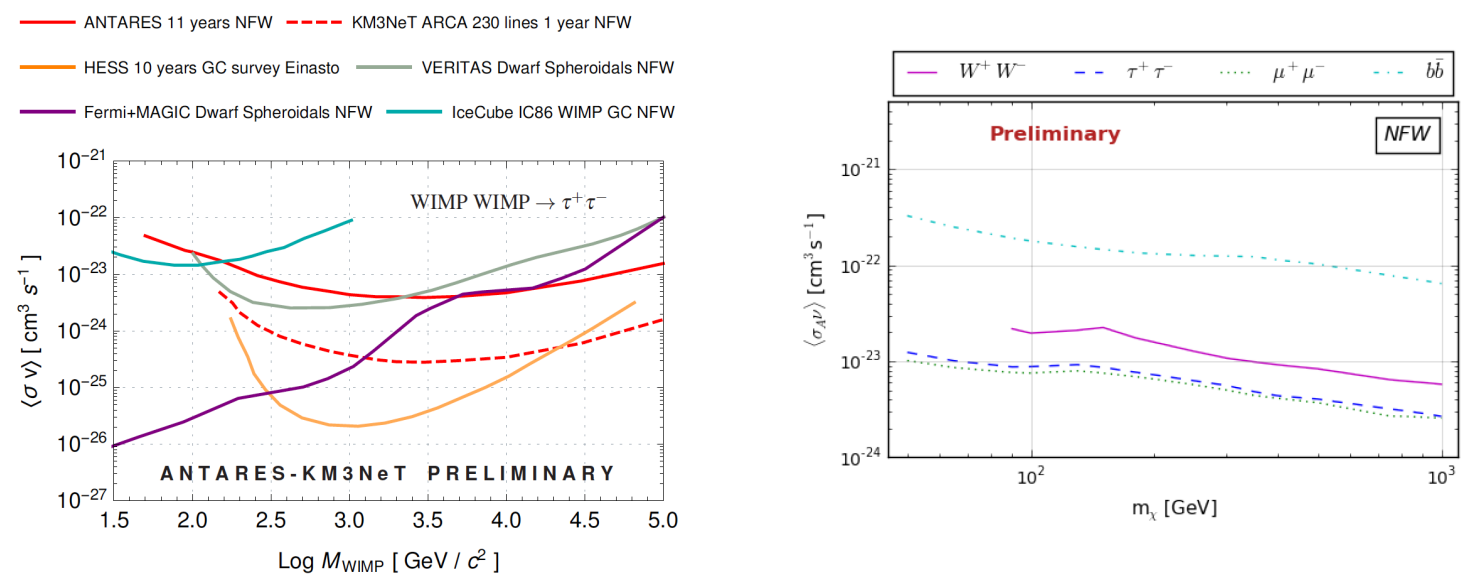

Figure 6: Left - Limits on the thermally averaged cross-section for WIMP pair annihilation set with 11 years of ANTARES data for the $\tau^{+} \tau^{-}$channel he obtained limits are also compared with current similar searches from IceCube and from $\gamma$-ray telescopes. The sensitivity for the KM3NeT/ARCA detector is also reported for 1 year of observation. Right - Limits on the thermally averaged cross-section for WIMP pair annihilation for the combined analysis ANTARES IceCube for all the channels analyzed.

\section{Neutrino oscillation studies}

The main aim of the KM3NeT/ORCA detector is the study of the fundamental properties of the neutrinos through the measurement of the oscillation of atmospheric neutrinos traversing the Earth. The expected performance for the measurements of the Neutrino Mass Ordering (NMO), the oscillation parameters $\Delta \mathrm{m}_{32}^{2}$ and $\sin ^{2} \theta_{23}$ and the normalization factor of the $v_{\tau}^{C C}$ appearance have been estimated [45] as well as the sensitivity for sterile neutrino [46] and Non-Standard Interactions (NSI) [47].

The energy range of interest for the NMO is between about 5-15 GeV [5]. In this region the oscillation probability, $\mathrm{P}\left(v_{x} \rightarrow v_{\mu}\right)\left(\mathrm{x}=v_{e}, v_{\mu}\right)$, for direct and inverse mass ordering reaches its maximal difference. The key ingredients related to the detector experimental features are the energy and zenith angular resolutions and the ability to distinguish the shower-like events from the track-like events. ORCA will provide a neutrino energy resolution of $\approx 25 \%$ and a zenith angle resolution of $\approx 5^{\circ}$ at $\mathrm{E}_{v}=10 \mathrm{GeV}$ for shower and track events. The identification between track and shower events is performed with a Random Decision Forest resulting in a correct identification of $80 \%$ of the track events and $90 \%$ for shower events. The analysis is based on pseudo experiments where the hypothesis of Normal Ordering (NO) was tested against the Inverse Ordering (IO) and vice versa. The calculation was done for $\delta_{C P}=0$ and $\delta_{C P}=\pi$ and for $\theta_{23} \in\left[40^{\circ}, 50^{\circ}\right]$. The oscillation parameters $\Delta \mathrm{m}_{21}^{2}$ and $\theta_{12}$ were fixed during fitting and $\theta_{13}$ was assigned as prior. The results reported in Fig. 7(left) for three years of observation show that the highest sensitivity (about $5 \sigma$ for $\theta_{23}=50^{\circ}$ ) for the rejection of the other ordering is seen if normal ordering is assumed with $\delta_{C P}=0$.

The sensitivity to $\Delta \mathrm{m}_{32}^{2}$ and $\sin ^{2} \theta_{23}$ has been also determined (for analysis details see [45]) for three years of observation with the ORCA full detector (115 DUs) and for one year of observation with the ORCA 7 DU detectors, that is planned to be deployed the next year. For comparison upper limits from other experiments are reported. After only three years of observation ORCA can 
constrain the $\Delta \mathrm{m}_{32}^{2}-\sin ^{2} \theta_{23}$ space significantly and even if with a small detector first additional inputs can be provided.

An interesting results is also the estimated sensitivity for the $v_{\tau}^{C C}$ normalization, $N_{v_{\tau}^{C C}}$. Deviation from $N_{v_{\tau}^{C C}}=1$ indicates a non unitarity of the $3 \times 3$ neutrino mixing matrix and then a hint for new physics or significant deviations from the predicted cross-section for charged-current $v_{\tau}$ interactions. The $v_{\tau}$ appearance, $\mathrm{P}\left(v_{\mu} \rightarrow v_{\tau}\right)$, experimentally is observed as an excess of shower like events for $\mathrm{E}_{v} \approx 20 \mathrm{GeV}$ and $\cos \theta<-0.5$. For ORCA 7 DUs $N_{v_{\tau}^{C C}}=0$ can be excluded at $3 \sigma$ significance after approximately 6 months of data taking [45].
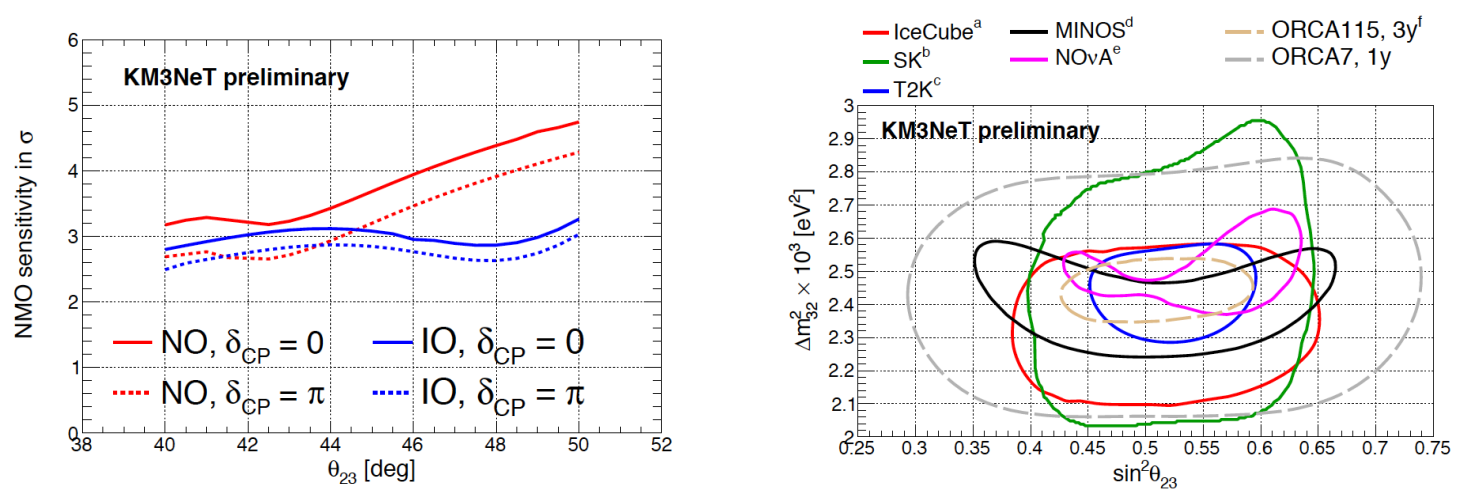

Figure 7: Left - Sensitivity for the NMO measurement of the KM3NeT/ORCA detector after 3 years of observation. Right - Sensitivity to $\Delta \mathrm{m}_{32}^{2}$ and $\sin ^{2} \theta_{23}$ the full ORCA detector after 3 years of observation and ORCA sub-array of 7 DUs after 1 year. For comparison upper limits from other experiments are reported.

A study to search for the existence of sterile neutrinos with ANTARES and the estimate of the expected sensitivity for ORCA has been presented in [46]. Significant distortions in the atmospheric neutrino oscillation pattern in the presence of light sterile neutrinos are expected. The main signal in the ANTARES and ORCA detectors is an expected excess of up-going track-like events in the energy range of $E_{v} \approx 20-30 \mathrm{GeV}$. Upper limits from ANTARES [48] and sensitivities from ORCA [46] to sterile neutrinos in the (3+1) model show that ANTARES has excluded values of the parameter space not yet excluded by other experiments and that the ORCA 115 DUs detector after three years of data taking can constrain significantly the present allowed phase space.

\section{KM3NeT status and first results}

Building on the experience gained with the ANTARES neutrino telescope and other projects in the Mediterranean Sea, the KM3NeT collaboration, after an R\&D program for the construction of innovative optical sensors and Detection Units, started to deploy the first strings on both ARCA and ORCA sites.

The first DU (DU1) of the ARCA detector was deployed in December 2015, followed by two in May 2016. One of the units deployed in May 2016 was recovered for inspection in July 2016. In the period May 2016 April 2017 the detector was operational with two DUs. Due to electrical problems in the network infrastructure at the seabed, the operations at the KM3NeT Italian site have been on hold since April 2017 and successfully restarted in January 2019 with only one DU (DU1) working. 
The first DU of ORCA (ORCA1) was installed in September 2017 and operated until midDecember 2017, when a failure of the main electro-optical cable made it impossible to power the underwater detector. After the replacement of a part in the main cable and two sea campaigns, the latest one in June 2019, 4 DUs are now being operated.

The first data collected has allowed checking the calibration procedures [49], testing the procedure to correct the data for the PMT inefficiency [50] and comparing MC simulations with the measured atmospheric muon events [51]. Moreover, even if data are collected with few strings it has been possible to select up-going neutrinos [52] among the large number of atmospheric muons. In this section some relevant results are reported.

The calibration of an instrument that cannot profit of calibrated sources or mono-energetic particle beams is a complex procedures. To achieve a nanosecond time accuracy, required to have a good angular resolution, the in situ and off-shore calibration procedure have been optimized. The description of the procedures are reported in Ref. [49].
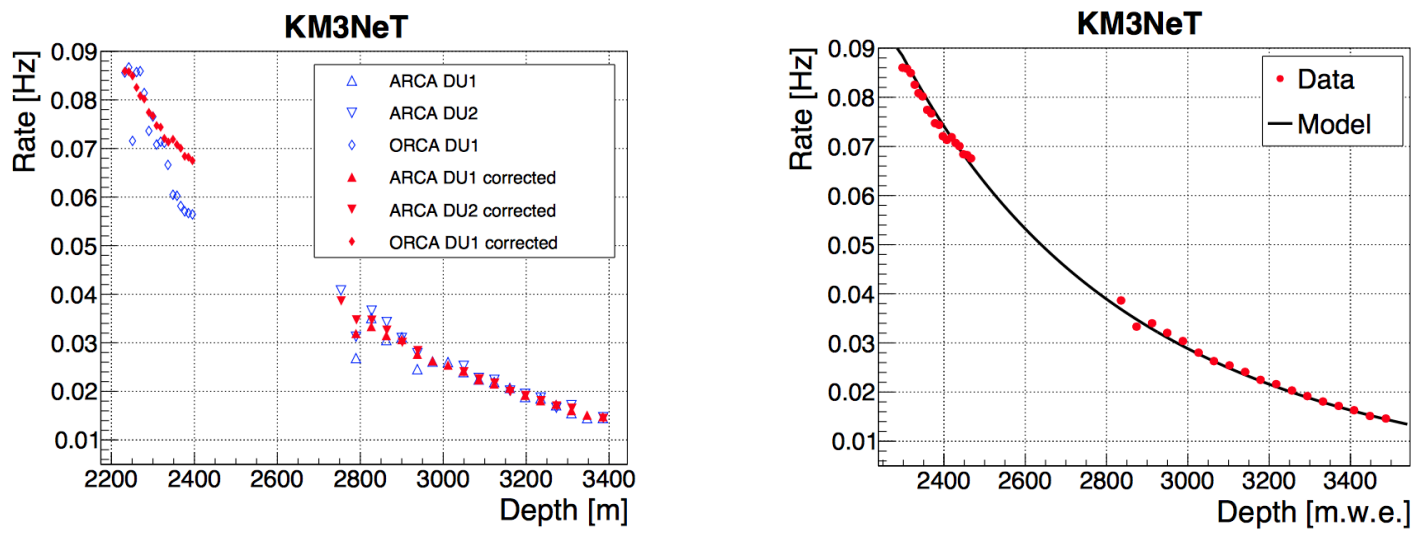

Figure 8: Multiplicity $\geq 8$ coincidence rate of all DOMs as function of depth below the sea level. Left -The coincidence rates for the ARCA2 and ORCA1 detectors are reported as measured (blue points) and after the correction for the PMT photon detection efficiencies (red points). Right- The corrected coincidence rate is reported as a function of the depth (red points) and fitted with the Bugaev model of the atmospheric muon flux (black line).

The features of a multi-PMTs sensor as the KM3NeT DOM (31 3-inch PMTs inside a glass sphere) were exploited measuring the atmospheric muon rate as a function of the sea depth [50]. The number of photons arriving in a single DOM together with the direction and the time information are used to discriminate signals from atmospheric muons and ${ }^{40} \mathrm{~K}$ backgrounds. The discriminating power is mainly achieved by exploiting coincidences between different PMTs in the same DOM. In this analysis, a coincidence is defined as a sequence of hits in a DOM within a time window of $15 \mathrm{~ns}$. The coincidence rate for multiplicity $M \geq 8$ (more than 8 PMTs with hits in coincidence in a DOM) is used to select a high-purity sample of atmospheric muons on a DOM-by-DOM basis [37]. In Fig. 8(left) the coincident rate as a function of the DOM depths for ORCA and ARCA before the correction (blue points) and after the correction for the PMT detection efficiency (red points) are reported. The depth range explored is about $1.2 \mathrm{~km}$. The PMT detection efficiency has been evaluated on a run-by-run basis. The corrected values are compared with the Bugaev [55] model predicting the depth dependence of atmospheric muons (Fig. 8(right)). 
The very good agreement (RMS of deviations within 2\%) proves that a single DOM is a "detector" able to distinguish muons from background and that the calibration procedure to estimate the PMT time offsets and PMT detection efficiency, based on coincidence hits from ${ }^{40} \mathrm{~K}$ decays, is verified.
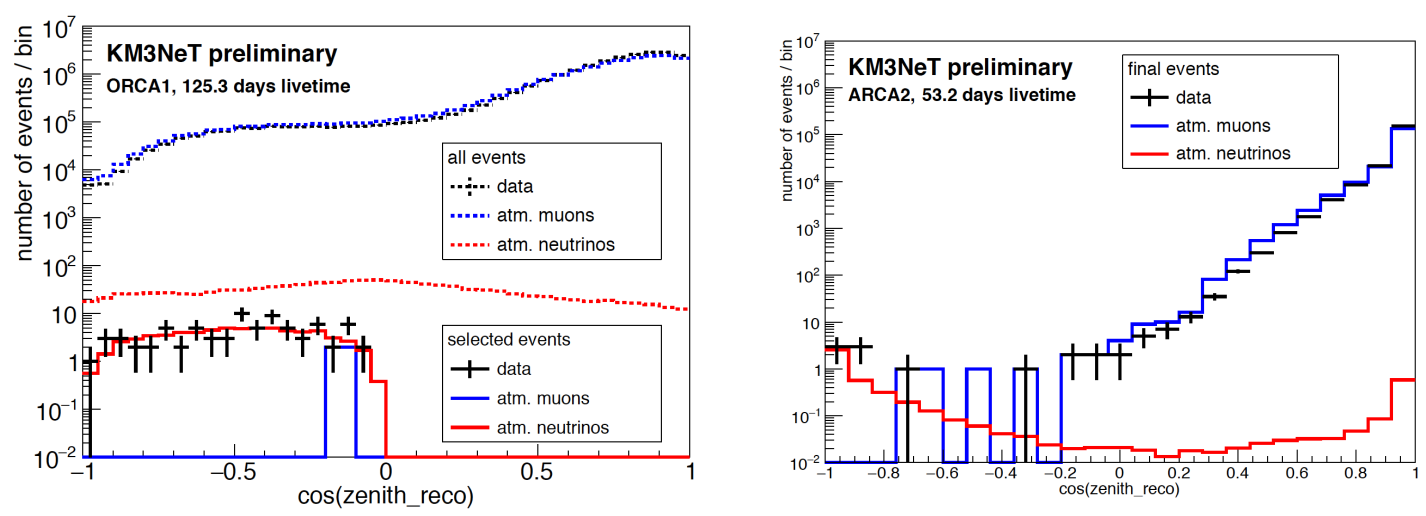

Figure 9: Reconstructed zenith distribution of ORCA1 events (left) and ARCA2 events (right).

The data collected with 2 DUs of ARCA (ARCA2) (livetime 53 days) and ORCA1 (livetime 123 days) were reconstructed with a track reconstruction algorithm and analyzed with the aim to select up-going neutrinos [52]. The reconstructed zenith angle is shown in Fig. 9(left) for the ORCA1 and right for ARCA2. The measured distribution in data is in good agreement with expectations from MC simulations. For ORCA1, 77 neutrino candidates are observed in the considered data sample, while 67 events are expected from atmospheric neutrino simulations and 4 events from atmospheric muon simulations. From the MC simulation has been shown that the selected neutrino events have an average energy of a few tens of GeV. With ARCA2, the search for atmospheric neutrinos is more challenging due to the higher neutrino energy detection threshold and the smaller effective livetime analyzed. For ARCA2 6 neutrino candidates with reconstructed $\cos \left(\theta_{\text {zenith }}\right)<0.8$ are found in the considered data sample while 3.3 events are expected from atmospheric neutrino simulations and 0 events from atmospheric muon simulations. The selected neutrino events show a typical energies of few hundreds of GeV. The results of this analysis have proven that even with a very low number of strings the selection of candidate neutrino events is possible.

\section{Conclusions}

Due to their extremely low interaction cross section, neutrinos can escape from dense source environments and, being not deflected from magnetic field, can reach the Earth preserving their original direction. Moreover, the simultaneous observation of GW [3] and neutrinos [1] with electromagnetic radiation has enforced the belief that only a detailed observation of all the cosmic messenger can shed light on the energetic non-thermal sources in the Universe.

The neutrino detectors located in the Mediterranean Sea can effectively contribute to a more precise observation of the high-energy neutrino flux thanks to their wide field of view and their very good angular resolution both for cascade and track events. ANTARES was the first undersea neutrino telescope constructed off shore the coast of Toulon (France) and it is taking data in its complete configuration from May 2008. A large variety of analyses has been carried out during these 
years spanning from neutrino oscillation studies to searches for neutrinos from different sources. In the next future to continue and improve the measurements of neutrinos the KM3NeT collaboration is constructing two neutrinos detectors in the Mediterranean Sea. With KM3NeT/ARCA a volume of about $1 \mathrm{~km}^{3}$ will be instrumented with a geometry that has been optimized for the detection of neutrinos of astrophysical origin. A denser instrumented detector, KM3NeT/ORCA, is being deployed off shore the Toulon coasts far about $10 \mathrm{~km}$ from the ANTARES detector, to study the neutrino oscillation properties. With these two detectors it will be possible to study neutrinos in a wide energy range from few $\mathrm{GeV}$ to $10^{8} \mathrm{GeV}$. In this work the most relevant results achieved by ANTARES after 11 years of data taking have been presented and the expectation for the next KM3NeT neutrino detectors also reported.

In the search for neutrinos from a diffuse flux the analysis adding two further years of data has reinforced the results presented in [19]. A mild excess of neutrinos above the background level has been observed with a significance of $1.8 \sigma$ (before $1.6 \sigma$ ). The estimated spectrum has a slope of $\Gamma=2.3_{-0.4}^{+0.4}$ and $\Phi_{0}(100 \mathrm{TeV})=1.5 \pm 1.010^{-18} \mathrm{GeV}^{-1} \mathrm{~cm}^{-2} \mathrm{~s}^{-1} \mathrm{sr}^{-1}$ and it is fully compatible with the IceCube measurements. The diffuse flux will be one of the first measurements that can be confirmed with a high significance by KM3NeT/ARCA. It was estimated that with the full detector the $5 \sigma$ level can be achieved after less than one year of observation.

The search for neutrinos from point-like sources was also addressed by ANTARES with several analyses. Even if no significant excess of events has been found, one interesting region of the sky has been put in evidence and studied more in detail. This region is located in the north sky at declination of $\delta=+23.6^{\circ}$ and $\alpha=343.5^{\circ}$ (full sky search). This point is coincident with the blazar MG3 J225517+2409. Around this source $\left(\delta=+24.19^{\circ}\right.$ and $\left.\alpha=343.78^{\circ}\right) 5$ ANTARES tracks lie at less than $1^{\circ}$ from the source. In addition, one IceCube high energy events was present within $1.1^{\circ}$ from the source center. The estimated post trial significance is of $1.4 \sigma$. A time dependent analysis for this source has provided a preliminary estimation of a pre-trial probability of about 2.2 $10^{-7}(5.2 \sigma)$ for the chance coincidence of observing such an association between ANTARES and IceCube neutrinos in space and time correlation with the blazar.

The discovery of Galactic neutrino sources can be addressed by KM3NeT/ARCA detector. Thanks to the high effective areas and the good visibility of the Galactic plane the discovery with a significance of about $3 \sigma$ of some of the most intense $\gamma$-sources can be achieved in few years.

ANTARES has also an intense activity in the multi-messenger physics, which will be further extended by KM3NeT. A real-time follow up program of the alerts from GCN is already well tested and also many analyses for counterpart searches have been already published.

KM3NeT is taking data with the first 4 DUs installed in the ORCA site and 1 DU in the ARCA site. Analyzing the first data has proved the robustness of procedures for the calibration of the detector, a validity of the simulation chain and in general a good understanding of the detector response.

ANTARES will continue data-taking until end of 2020 when KM3NeT will start to have a significant instrumented volume thus assuring a continue monitoring of the neutrino sky from the Northern hemisphere. 


\section{References}

[1] The IceCube, Fermi-LAT, MAGIceCube, AGILE, ASAS-SN, HAWC, H.E.S.S, INTEGRAL, Kanata, Kiso, Kapteyn, Liverpool telescope, Subaru, Swift/NuSTAR, VERITAS, and VLA/17B-403 teams, Science 361, eaat1378 (2018).

[2] Aartsen M.G. et al. (IceCube Coll.) Science 361, 147-151 (2018).

[3] Abbott B.P. et al., The Astrophysical Journal Letters, 848, Number 2 (2017).

[4] Ageron A. et al., (ANTARES coll.) Nucl. Instrum. Meth. A 65611 (2011).

[5] Adrian-Martinez S. et al., (KM3NeT coll.) Journal of Physics G: Nuclear and Particle Physics, 43 084001 (2016).

[6] Albert A. et al. (ANTARES Coll.) Phys. Rev. D 96082001 (2017).

[7] ANTARES Coll. PoS(ICRC2019)920.

[8] Albert A. et al., (ANTARES Coll.) ApJL 863, L30 (2018).

[9] ANTARES Coll. PoS(ICRC2019)840

[10] Aartsen M.G. et al. (IceCube Coll.)Astrophys. J. 8333 (2016).

[11] Aartsen M.G. et al. (IceCube Coll.) Astrophys. J. 8352 (2017).

[12] ANTARES and IceCube Coll. PoS(ICRC2019)919.

[13] Ackermann M., et al., (Fermi-LAT Collaboration) it Science 339807 (2013).

[14] Aiello S. et al., (Km3NeT Coll.) it Astroparticle Physics 111100 (2019).

[15] Aartsen M.G. et al. (IceCube Coll.) Astrophys. J. 80998 (2015).

[16] IceCube Coll. POS(ICRC2017)981.

[17] Murase K. and Eli Waxman E. Phys. Rev. D 94103006 (2016).

[18] Palladino A. and Winter W. A.A. A168 615 (2018).

[19] Albert A. et al. (ANTARES Coll.) The Astrophysical Journal Letters, 853, Number 7 (2018).

[20] ANTARES Coll. PoS(ICRC2019)891.

[21] Honda M. et al., Phys. Rev. D 75043006 (2077).

[22] Enberg R., Reno M.H. and Sarcevic I. Phys. Rev. D 78043005 (2008).

[23] Icecube Coll. PoS(ICRC2017)1005.

[24] Albert A.et al., Phys. Rev. D 96062001 (2017).

[25] Albert A. et al. (ANTARES and IceCube Collaborations), Astrophys.J. 868 L20 (2018)

[26] Gaggero D., Grasso D., Marinelli A., Taoso M. and Urbano A. Phys Rev. Lett., 119031101 (2017).

[27] Andersen K.J. et al., The Astrophysical Journal Letters, 861, L19 (2018).

[28] ANTARES Coll. PoS(ICRC2019)892.

[29] KM3NeT Coll. PoS(ICRC2017)998.

[30] KM3NeT Coll. PoS(ICRC2017)870. 
[31] Ageron M. et al. (ANTARES Coll.) Astropart.Phys. 35 5530-536 (2012).

[32] ANTARES Coll. PoS(ICRC2019)871.

[33] ANTARES Coll. PoS(ICRC2019)872.

[34] ANTARES, IceCube and LIGO-Virgo Coll. Phys Rev. D., 93122010 (2016). ANTARES, IceCube and LIGO-Virgo Coll. Phys Rev. D., 96022005 (2017), Albert A. et al. (ANTARES Coll.) Eur. Phys, J. C 91177 (2017). ANTARES Coll. (PoSIceCubeRC2019)856.

[35] ANTARES Coll. PoS(ICRC2019)984.

[36] KM3NeT Coll. PoS(ICRC2019)857

[37] Adrian-Martinez S. et al., (KM3NeT Coll. ) Eur. Phys. J. C 76:54 (2016).

[38] ANTARES and KM3NeT Coll. PoS(ICRC2019)552

[39] Cirelli M et al., JCAP 1103051 (2011). [Erratum: JCAP1210,E01(2012)].

[40] Aartsen M. G. et al. (IceCube Coll.), Eur. Phys. J. C 77.627 (2017).

[41] ANTARES Coll. PoS(ICRC2019)522

[42] Albert A. et al. (ANTARES Coll.), Phys. Lett. B 769249 (2017). Erratum: Phys.Lett.B (2019)

[43] Adrian-Martinez S. et al., (ANTARES Coll. ) Phys. Lett. B 759 69-75 (2016).

[44] KM3NeT Coll. PoS(ICRC2019)536

[45] KM3NeT Coll. PoS(ICRC2019)1019

[46] KM3NeT Coll. PoS(ICRC2019)870

[47] KM3NeT Coll. PoS(ICRC2019)931

[48] Albert A. et al., (ANTARES Coll.), J. High Energ. Phys. 06113 (2019).

[49] KM3NeT Coll. PoS(ICRC2019)932.

[50] Ageron M. et al., (KM3NeT Coll.) https://arxiv.org/pdf/1906.02704.pdf and KM3NeT Coll. PoS(ICRC2019)943.

[51] KM3NeT Coll. PoS(ICRC2019)927.

[52] KM3NeT Coll. PoS(ICRC2019)910.

[53] Carminati M. et al., Comp. Phys. Comm. 179915 (2008).

[54] CORSIKA web page. Available on http://www.ikp.kit.edu/corsika/.

[55] Bugaev E.V. et al., Phys.Rev.D 58054001 (1998). Klimushin S.I. et al., Phys. Rev. D 64014016 (2001). 